\title{
On limit multiplicities of discrete series representations in spaces of automorphic forms
}

\section{Laurent Clozel*}

Department of Mathematics, University of Michigan, Ann Arbor, MI 48109, USA

\section{Introduction}

Let $G$ be a connected semisimple real Lie group, $\Gamma$ an arithmetic subgroup of $G$. Assume $G$ has a discrete series of representations. We will say that a sequence $\Gamma_{n}$ is a tower of subgroups of $\Gamma$ if $\left[\Gamma: \Gamma_{n}\right]$ is finite for all $n$, and

$$
\lim _{n \rightarrow \infty}\left[\Gamma: \Gamma_{n}\right]=\infty .
$$

Of course the groups $\Gamma_{n}$ are then arithmetic subgroups of $G$. In this situation, it seems natural to assume that, as $n \rightarrow \infty$, the spectral decomposition of $L^{2}\left(\Gamma_{n} \backslash G\right)$ will approximate that of $G$. In particular, let $\delta$ be a discrete series representation of $G$. If $\Gamma$ is a subgroup of $G$, let $m(\Gamma, \delta)$ be the multiplicity of $\delta$ in the representation of $G$, by right translations, on $L^{2}(\Gamma \backslash G)$. Choose a Haar measure $d g$ on $G$; if $\Gamma$ is arithmetic, let $v(\Gamma \backslash G)$ be the (finite) volume of $\Gamma \backslash G$ for the measure induced by $d g$. Let $d(\delta)$ be the formal degree of $\delta$ for the measure $d g$.

Conjecture $A$. Let $\left(\Gamma_{n}\right)$ be a tower of subgroups of $\Gamma$. Then

$$
\lim _{n \rightarrow \infty} \frac{m\left(\Gamma_{n}, \delta\right)}{v\left(\Gamma_{n} \backslash G\right)}=d(\delta) .
$$

When $\Gamma \backslash G$ is compact, this conjecture has been proved by DeGeorge and Wallach [20]. (Note that $\Gamma$ does not have to be assumed arithmetic in that special case.) In the general case, the Conjecture should follow some day from a better understanding of the Arthur-Selberg Trace Formula. Partial results when the real rank of $G$ is 1 and $\delta$ is regular enough - have been obtained by DeGeorge [19] and Barbasch-Moscovici [8]. Meanwhile, and in view of the applications, it seems of interest to prove a weaker result, which still implies that the representation $\delta$ imbeds, with large enough multiplicity, in spaces of automorphic forms. Kazhdan announced in [26] a proof of the following:

* Partially supported by NSF Grant MCS-8211506 
Conjecture $B$. Under the same assumptions, there exists $\varepsilon>0$ such that

$$
\liminf _{n \rightarrow \infty} \frac{m\left(\Gamma_{n}, \delta\right)}{v\left(\Gamma_{n} \backslash G\right)}>\varepsilon .
$$

In this article we will prove a weak version of Conjecture B for congruence subgroups. Let $G$ be a connected, simply connected, semi-simple, almost simple group defined over $\mathbb{Q}$. We assume that $G=\underline{G}(\mathbb{R})$ is noncompact. Let $\Gamma$ be a congruence subgroup of $G$. If $S$ is a finite set of primes, we define in $\S 2$ the (natural) notion of "a sequence of subgroups $\Gamma_{n}$ of $\Gamma$ tending to 1 at $S$ ". If $\Gamma_{n}$ is

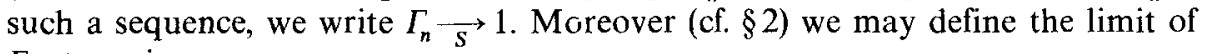
$\Gamma_{n}$ at a prime $p$.

Assume now that $p_{0}$ is a prime such that $\underline{G}\left(\mathbb{Q}_{p_{0}}\right)$ has a supercuspidal representation.

Theorem 1. Let $\Gamma \subset G$ be a congruence subgroup. Then there is a subgroup $\Gamma_{0}$ of $\Gamma$ such that, if $S$ is a finite set of primes not containing $p_{0}$, and $\Gamma_{n}$ is a sequence of subgroups of $\Gamma_{0}$ such that $\Gamma_{n} \vec{S} 1$ :

$$
\liminf _{n \rightarrow \infty} \frac{m\left(\Gamma_{n}, \delta\right)}{v\left(\Gamma_{n} \backslash G\right)}>\varepsilon>0 .
$$

Here $\varepsilon$ is a constant depending only on $G, \Gamma_{0}$ and the limit of the sequence $\left(I_{n}\right)$ at $p_{0}$.

As may be seen, Theorem 1 is enough to complete Kazhdan's proof of his theorem on the conjugates of arithmetic varieties by automorphisms of $\mathbb{C}$, when the variety is not complete $[27, \S 5]$. It also has obvious consequences for the cohomology of arithmetic groups and the $L^{2}$-cohomology of arithmetic manifolds. If $\Gamma_{n}$ is a tower of congruence subgroups of $G$ as in Theorem 1, the cohomology of $\Gamma_{n}$ in the middle dimension will grow at least as the covolume. Also, our result joined to work of Borel and Casselman [12] shows that the criterion they obtained for the finite-dimensionality of the $L^{2}$-cohomology of $\Gamma \backslash G / K$ ( $K$ maximal compact in $G$ ) is necessary - for $\Gamma$ deep enough - as well as sufficient. These consequences are detailed in $\S 5$. Also, in $\S 4$, we give a theorem realizing discrete series representations of $p$-adic groups in spaces of cusp forms.

The proof relies on three main ingredients. The first one is Arthur's version of the Trace Formula for adèle groups (for an introductory account see [28]). This gives a formula for the trace of a function $f$ on the adelic group acting in the discrete spectrum of automorphic forms, in terms of certain distributions containing in particular orbital integrals of $f$. If one wants to prove Conjecture $\mathrm{A}$, the only important term is a multiple of $f(1)$. Thus easy versions of the trace formula, from which this term is missing, are of no avail. Even to prove our weaker theorem, one needs a recent result of Arthur giving a fairly manageable expression for the term associated to unipotent orbital integrals.

The second ingredient is a theorem of Delorme and the author $[16,17]$, constructing pseudo-coefficients on a real semi-simple group. This allows us to eliminate, from the "representation-theoretic" side of the trace formula, all 
terms except those relative to a finite number of representations of $G$. The third is a method introduced by DeGeorge and Wallach which shows that the terms pertaining to representations other than $\delta$ vanish at the limit.

The article is organized as follows. In $\$ 2$ we recall some definitions and results on arithmetic subgroups of semi-simple groups. In $\S 3$, we prove the adèlic version of Theorem 1 . In $\S 4$, we give an extension of the adelic theorem to $p$-adic places. The theorem itself is deduced in $\S 5$, as well as the corollaries concerning cohomology.

\section{Preliminaries}

2.1. Let $G$ be a connected real semi-simple Lie group. We will use the extended notion of arithmetic subgroups of $G$, which we now recall. We refer, e.g., to [30].

If $G=G(\mathbb{R})^{+}$, the connected component of the real-valued points of a group $\underline{G}$ defined over $\mathbb{Q}$, a subgroup $\Gamma$ of $G$ is strongly arithmetic if there is an embedding $\psi$ of $\underline{G}$ into $G L(R)$, defined over $\mathbb{Q}$, such that $\psi(\Gamma)$ is commensurable with $G \cap G L(R, \mathbb{Z})$. The group $\Gamma$ is a congruence subgroup if there is such an imbedding for which $\psi(\Gamma) \supset G \cap \Delta$, where $\Delta$ is a congruence subgroup of $G L(R, \mathbb{Z})$.

If now $G$ is just a connected semi-simple Lie group, a subgroup $\Gamma$ of $G$ is arithmetic if there is a semi-simple group $G_{1}$ defined over $\mathbb{Q}$ and a surjective homomorphism $\varphi: \underline{G}_{1}(\mathbb{R})^{+} \rightarrow G$ with compact kernel such that the image of a strongly arithmetic subgroup of $G_{1}(\mathbb{R})^{+}$is commensurable with $\Gamma$.

2.2. Assume now that $\underline{G}$ is simply connected, so that $\underline{G}(\mathbb{R})$ is connected. Let $G(\mathbb{A})$ be the group of $\mathbb{A}$-valued points of $G$, where $\mathbb{A}$ is the ring of adèles of $\mathbb{Q}$. Then congruence subgroups are just groups of the form $\underline{G}(\mathbb{Q}) \cap(\underline{G}(\mathbb{R}) \times K), K$ being a compact-open subgroup of $\underline{G}\left(\mathbb{A}_{f}\right), \mathbb{A}_{f}$ the finite adèles.

If moreover $G$ has the strong approximation property - in particular if $G$ is simply connected, almost simple over $\mathbb{Q}$, and $G(\mathbb{R})$ is noncompact - there is a natural bijection between $G(\mathbb{Q}) \backslash G(\mathbb{A}) / K$ and $\Gamma \backslash G(\mathbb{R}), \Gamma$ being the congruence subgroup defined by $K$. This will be used in $\S 5$ to compare "classical" and "adèlic" automorphic forms (cf. Borel-Jacquet [13]).

2.3. Assume $G$ is as in the previous paragraph, and satisfies in particular the strong approximation property. If $\Gamma$ is a congruence subgroup of $G$, let $K_{r}$ be the associated subgroup of $G\left(\mathbb{A}_{f}\right)$. If $\Gamma_{n}$ is a sequence of congruence subgroups of $\Gamma$, and $S$ a finite set of primes, we say that $\Gamma_{n} \rightarrow 1$ at $S$ if, setting $K_{n}=K_{\Gamma_{n}}$ :

For all large enough $n, K_{n}$ is a direct product $K_{n}=K_{S, n} \times K^{S}$, with $K^{S}$ $=\prod_{p \notin S} K_{p}$ a product of local groups, independent of $n$, and $K_{S, n} \underset{n \rightarrow \infty}{\longrightarrow} 1$.

(Note that we assume rather stringent properties of the decomposition of $K_{\Gamma_{n}}$ as a product.)

If $p \notin S, K_{p}$ is called the limit of the sequence $\Gamma_{n}$ at $p$. 


\section{The adèlic theorem}

3.1. We consider a semi-simple connected group defined over $\mathbb{Q}$. In this paragraph, we denote it by $G$ instead of $G$; then $G_{\infty}=G(\mathbb{R}), G_{p}=G\left(\mathbb{Q}_{p}\right), G(\mathbb{A})$ denote the points with values in $\mathbb{R}, \mathbb{Q}_{p}$ and $\mathbb{A}$. We assume that $G_{\infty}$ has a discrete series.

We choose a prime $p_{0}$ such that the group $G_{p_{0}}$ has supercuspidal representations. Using the methods in Gérardin [21, Chap. 5], such representations may be constructed if $G$ is split, and thus a Chevalley group, at $p_{0}$, and if the associated finite reductive group has cuspidal representations; thus it is true at least for a set of primes having non-zero density, thanks to the results of Deligne and Lusztig for finite groups.

We choose such a representation $\pi_{0}$ of $G_{p_{0}}=G_{0}$. Let $K_{p_{0}}=K_{0}$ be a compact-open subgroup of $G_{0}$ such that $\pi_{0}$ contains non-zero vectors fixed by $K_{0}$. Moreover, let $f_{0}$ be the function defined on $G_{0}$ by $f_{0}(g)=\langle g w, w\rangle$ for such a vector $w$. We assume that $\langle w, w\rangle=1$. Then, by Schur's orthogonality relations [24], trace $\pi_{0}\left(f_{0}\right)=d\left(\pi_{0}\right)^{-1}$. (We choose a Haar measure $d g_{\mathbb{A}}=\underset{v}{\bigotimes} d g_{v}$ on $G(\mathbb{A})$; both the trace and the formal degree $d\left(\pi_{0}\right)$ are then computed by means of $\left.d g_{0}\right)$.

Let $S$ be a finite set of primes such that $p_{0} \notin S$. Let $K_{S}$ be a compact-open subgroup of $G_{S}=\prod_{p \in S} G_{p}$. If $K_{S}$ is a variable such subgroup, defined, say, by a directed set of indices, we say that $K_{S} \rightarrow 1$ if, for any compact-open subgroup $U$ of $G_{S}$ the subgroup $\left(K_{S}\right)_{i}$ is contained in $U$ for large enough $i$.

Let $\mathscr{L}=L_{\text {cusp }}^{2}(G(\mathbb{Q}) \backslash G(\mathbb{A}))$ be the space of cusp forms on $G(\mathbb{Q}) \backslash G(\mathbb{A})$. If $K$ is a compact-open subgroup of $G\left(\mathbb{A}_{f}\right)$, we write $\mathscr{L}^{K}$ for the space of $K$ invariants (by the natural right-action) in $\mathscr{L}$. It is still a $G_{\infty}$-module.

Finally, let us choose a compact-open subgroup $K^{S, p_{0}}$ of

Theorem 1 A.

$$
G^{S, p_{0}}=\prod_{p \notin S \cup\left\{p_{0}\right\} \cup \infty} G_{p} .
$$

$$
\begin{gathered}
\liminf _{K_{S} \rightarrow 1}\left[v\left(K_{S}\right) \operatorname{mult}\left(\delta, L_{\text {cusp }}^{2}(G(\mathbb{Q}) \backslash G(\mathbb{A}))^{K_{0} \times K_{S} \times K^{S, p_{0}}}\right)\right] \\
\geqq v(G(\mathbb{Q}) \backslash G(\mathbb{A})) \cdot d\left(\pi_{0}\right) \operatorname{dim}\left(\pi_{0}^{K_{0}}\right) d(\delta) v\left(K^{S, p_{0}}\right)^{-1}
\end{gathered}
$$

An extension of Theorem 1A to certain discrete series representations of $G_{S}$, where $S$ is a finite set of primes, will be given in the next paragraph.

3.2. The proof will be obtained by applying Arthur's trace formula $([1,2]$; see also [18]) to a suitable function $f$ on $G(\mathrm{~A})$. For simplicity we first assume that $K^{S, p_{0}}$ is a product of local groups; this condition will be removed in $\S 3.4$.

We take $f$ to be a tensor product $f=f_{\infty} \otimes f_{0} \otimes f_{S} \otimes \otimes f_{p}$.

$$
\underset{p}{\text { remaining }}
$$

The function $f_{0}$ is chosen as above. For given $K_{S}$, let $h_{S}$ be the characteristic function of $K_{S}$, we set $f_{S}=\operatorname{vol}\left(K_{S}\right)^{-1} h_{S}$ : thus $f_{S}$ depends, in fact, on $K_{S}$, and, 
for the convolution defined by the measure $d g_{S}$, it satisfies $f_{S} * f_{S}=f_{S}$. For other finite primes $p$, define $f_{p}$ in the same manner, using the fixed group $K_{p}$; of course, $\operatorname{vol}\left(K_{p}\right)=1$ for almost all $p$.

We still have to define $f_{\infty}$. Let $K_{\infty}$ be a maximal compact subgroup of $G_{\infty}$. By Theorem 1.1 of [16], there is a smooth, compactly supported function $f_{\infty}$ on $G_{\infty}, K_{\infty}$-finite on both sides, such that trace $\delta\left(f_{\infty}\right)=1$, and trace $\pi\left(f_{\infty}\right)=0$ for any tempered irreducible representation $\pi \neq \delta$ of $G$. This is our choice for $f_{\infty}$. (Such a function is called a pseudo-coefficient of $\delta$.)

Lemma 1. There exist only a finite number of irreducible representations $\pi$ of $G_{\infty}$ (up to isomorphism) such that trace $\pi\left(f_{\infty}\right) \neq 0$. Moreover, none of them except $\delta$ belong to the discrete series.

Proof. By a theorem of Harish-Chandra and Zuckerman, any $\pi$ can be uniquely written as a sum, with integral coefficients, of basic representations. It is shown in $[16,17]$ that the trace of $f_{\infty}$ in any basic representation except $\delta$ is 0 . But then, if trace $\pi\left(f_{\infty}\right) \neq 0$, the expression of $\pi$ must involve $\delta$; this implies that the infinitesimal character of $\pi$ is that of $\delta$, whence the first assertion. This also shows that $\pi$ cannot be a basic representation - and à fortiori a discrete series representation - unless $\pi=\delta$.

We now apply Arthur's trace formula. It is expressed by an identity [2, p. 120]

$$
\sum_{\mathbf{o} \in \mathfrak{C}} J_{\mathscr{O}}^{T}(f)=\sum_{\chi \in \mathscr{X}} J_{\chi}^{T}(f)
$$

of certain distributions evaluated at $f$. Here $T$ is a suitable regular point in $\mathfrak{a}_{0}$, the real Lie algebra of the split component of a minimal Levi subgroup of $G$ defined over $\mathbb{Q}$. We explain the right-hand side first.

Lemma 2. For $f$ as above, $\sum_{\chi \in \mathscr{X}} J_{\chi}^{T}(f)$ is the trace trace $r_{\text {cusp }}(f)$ of $f$ acting by convolution on the space $L_{\text {cusp }}^{2}(G(\mathbb{Q}) \backslash G(\mathbb{A}))$; it does not depend on $T$.

Proof. This could be proved rather directly, tracing the effect of truncation by $T$ on functions. We prefer, for brevity, to use Arthur's end results.

The set $\mathscr{X}$ is the set of cuspidal data, i.e., pairs $(M, \rho)$ where $M$ is a Levi subgroup of $G$ over $\mathbb{Q}$, and $\rho$ an irreducible, cuspidal representation of $M(\mathbb{A})^{1}$, the subgroup of elements of $M(\mathbb{A})$ on which all rational characters have absolute value 1 . See $[1, \S 3]$ or $[18$, Lecture 1$]$; data conjugate by the inner action of $G$ are identified.

The definition of $J_{\chi}^{T}(f)$ is given in [2, p. 88] and an expression for it is obtained in $[2$, p. 120]:

$$
J_{\chi}^{T}(f)=\sum_{P} n(A)^{-1} \int_{\Pi(M)} \operatorname{trace}\left(M_{P}^{T}(\pi)_{\chi} I_{P}(\pi, f)_{\chi}\right) d \pi .
$$

Here the sum is over all parabolic subgroups $P=M N$ containing a given minimal parabolic subgroup $P_{0} ; n(A)^{-1}$ is a certain constant; $\Pi(M)$ can be taken to be the set of isomorphism classes of representations of $M(\mathbb{A})$ whose restriction to $M(\mathbb{A})^{1}$ occurs discretely in $L^{2}\left(M(\mathbb{Q}) \backslash M(\mathbb{A})^{1}\right)([1, \S 3]$; note that $G$ 
is semi-simple; also, Arthur defines $\Pi(M)$ as the set of all isomorphism classes, but the term associated to $\pi$ is then 0 unless $\pi$ occurs discretely). For given $\pi \in \Pi(M), I_{P}(\pi)$ is the representation of $G(\mathbb{A})$ induced from the representation $\sigma \otimes 1$ of $P(\mathbb{A})=M(\mathbb{A}) N(\mathbb{A})$, where $\sigma$ is the subspace of the discrete spectrum (modulo the center) of $M(\mathrm{~A})$ spanned by representations isomorphic to $\pi$; $I_{p}(\pi, f)_{\chi}$ is the operator given by convolution by $f$ in the subspace of $I_{P}(\pi)$ given by residues of Eisenstein series built from $\chi$; finally, $M_{p}^{T}(\pi)$ is a certain operator in the space of $I_{P}(\pi, f)_{\chi}$ associated to truncation at the parameter $T$.

Now, since $f_{0}$ is a coefficient of a supercuspidal representation, the local operator $I_{p}\left(\pi, f_{0}\right)$ is zero for any representation $\pi$ of $M\left(\mathbb{Q}_{p_{0}}\right)$ if $P$ is a proper subgroup. (This is well known; for a proof, see Henniart [25, Appendix 1]). Since the global operators $I_{P}(\pi, f)$ are tensor products of local operators, we see that only the terms with $P=G$ in the expression of $J_{x}^{T}(f)$ are non-zero. Thus

$$
J_{\chi}^{T}(f)=\int_{\Pi(G)} \operatorname{trace}\left(M_{G}^{T}(\pi)_{\chi} I_{G}(\pi, f)_{\chi}\right) d \pi
$$

The integral is now in fact a discrete sum. Assume now that the datum $\chi$ is a pair $(\rho, M)$ with $M \neq G$. By definition $I_{G}(\pi, f)_{\chi}$ is the subspace of $L_{\mathrm{dis}}^{2}(G(\mathbb{Q}) \backslash G(\mathbb{A}))$ composed of representations of type $\pi$, obtained from Eisenstein series associated to functions in the space of $L_{\text {cusp }}^{2}\left(M(\mathbb{Q}) \backslash M(\mathbb{A})^{1}\right)$ of type $\rho$. If $\tau$ is an irreducible representation of $G$ contained in $I_{G}(\pi, f)_{\chi}$, Eisenstein series and their residues (cf. e.g., Arthur [3]) provide an intertwining operator from $I_{P}\left(\tau_{M}\right)$ onto $\tau$, where $\tau_{M}$ is a cuspidal (non-unitary) representation of $M(\mathbb{A})$ such that $\left.\tau_{M}\right|_{M(\mathbf{A})^{1}} \cong \rho$. But again, by the condition on $f_{0}$, we have $I_{P}\left(\tau_{M}, f\right)=0$ whence $I_{G}(\pi, f)_{\chi}=0$. Thus we are left with the terms cuspidal for $G$, which proves Lemma 2.

We now explain the left-hand side of $(*)$. Here the sum runs over the set $\mathcal{O}$ of $G(\mathbb{Q})$-conjugacy classes of semi-simple elements of $G(\mathbb{Q})$. If $o \in \mathcal{O}$, Arthur defines $([1$, p. 947, 942])

$$
J_{\mathbf{v}}^{T}(f)=\int_{G(\mathbb{Q}) \backslash G(\mathbb{A})} k_{\mathbf{o}}^{T}(x, f) d x
$$

where

$$
k_{\mathrm{o}}^{T}(x, f)=\sum_{P}(-1)^{a_{P}} \sum_{\delta \in P(\mathbb{Q}) \backslash G(\mathbb{Q})} K_{P, \mathbf{v}}(\delta x, \delta x) \cdot \hat{\tau}_{P}(H(\delta x)-T),
$$

([1, p. 938]). Here the sum runs over all standard parabolic subgroups $P$ $=M N ; a_{P}$ is the dimension of the split component of $M ; \hat{\tau}_{P}(H(\delta x)-T)$ is, for $\delta$ and $x$ given, a real number whose definition need not concern us (cf. [1, p. 936]); if $P=G$, it is identically equal to 1 . Lastly,

$$
K_{P, \mathbf{0}}(x, y)=\sum_{\gamma \in M(\mathbb{Q}) \cap \mathbb{O}} \int_{\mathbb{N}(\mathbf{A})} f\left(x^{-1} \gamma n y\right) d n
$$

for $x, y \in N(\mathbb{A}) M(\mathbb{Q}) \backslash G(\mathbb{A})$. (See $[1$, p. 923].) 
Lemma 3. For $f$ as above,

$$
J_{\mathbf{0}}^{T}(f)=\int_{G(\mathbb{Q}) \backslash G(\mathbb{A})}\left\{\sum_{\gamma \in \mathbf{D}} f\left(x^{-1} \gamma x\right)\right\} d x ;
$$

the integral $\int\left|\sum_{\gamma \in \mathcal{O}} f\left(x^{-1} \gamma x\right)\right| d x$ is absolutely convergent.

Proof. Arthur shows (Thm. 7.1) that $\int\left|k_{v}^{T}(x, f)\right|$ is absolutely convergent, so we only have to prove that

$$
k_{\mathbf{v}}^{T}(x, f)=\sum_{\gamma \in \mathbf{0}} f\left(x^{-1} \gamma x\right) .
$$

(Such a sum is finite.) This means that, in the expression of $k_{\mathfrak{v}}^{T}$ given above, all terms $K_{P, 0}$ for $P \neq G$ vanish. But if $P \neq G$ we have $\int_{N\left(\mathbb{Q}_{P_{0}}\right)} f_{0}\left(x^{-1} \gamma n y\right) d n=0$ for any $x, \gamma, y$ by a fundamental property of coefficients of supercuspidal representations (cf. e.g., [22, Thm.6]). A fortiori, the global integral vanishes, whence the result.

At this point, we have proved:

Lemma 4. For $f$ as above,

Moreover,

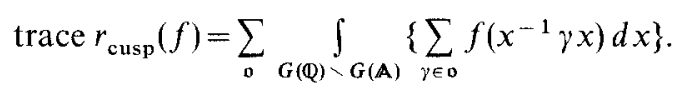

$$
\sum_{\mathbf{0}} \int\left|\sum_{\gamma \in \mathbf{D}} f\left(x^{-1} \gamma x\right)\right|<\infty
$$

Again, the convergence assertion is contained in [1, Thm. 7.1].

Lemma 5. For $K_{S}$ small enough, $f\left(x^{-1} \gamma x\right)=0$ for any $x \in G(\mathbb{A})$, unless $\gamma$ is unipotent.

Proof. Using a faithful representation: $G \rightarrow G L(R)$, defined over $\mathbb{Q}$, we may just identify $G$ to a subgroup of $G L(R)$. For $x \in G$, let

$$
p(x, T)=\operatorname{det}(x-(1-T))=T^{R}+a_{1}(x) T^{R-1}+\ldots+a_{R}(x) ;
$$

the $a_{i}$ are then polynomial functions on $G$, defined over $\mathbb{Q}$. In particular, for $x \in G(\mathbb{A}), a_{i}(x) \in \mathbb{A}$ is well-defined. Moreover,

$$
a_{i}(x)=\left\{a_{i}\left(x_{\infty}\right) a_{i}\left(x_{0}\right) \prod_{\substack{p \notin S \\ p \neq p_{0}}} a_{i}\left(x_{p}\right)\right\} \prod_{p \in S} a_{i}\left(x_{p}\right) .
$$

When $K_{S}$ varies, and $f$ varies accordingly, the bracketed term is constant and bounded independently of $x$ in the support of $f$, since the support of $f_{v}$ for $v \notin S$ does not vary. Let $|x|_{p}$ be the normalized $p$-adic absolute value of $x \in \mathbb{Q}_{p}$; if $x \in \mathbb{A}$, let $|x|=\prod_{v}\left|x_{v}\right|_{v}$ be its absolute value. 
Taking $K_{\mathrm{S}}$ small enough, we may arrange that, on the support of $f, \mid a_{i}\left(x_{p}\right)$ $-\left.a_{i}(1)\right|_{p}=\left|a_{i}\left(x_{p}\right)\right|_{p}<\varepsilon$ for any given $\varepsilon$. Taking the product of the local valuations, we see that for small enough $K_{S}$, we may arrange:

$$
\left|a_{i}(x)\right|<1 \quad(x \in \operatorname{Supp}(f)) .
$$

But now, if $f\left(x^{-1} \gamma x\right)=0, \gamma$ is conjugate in $G(\mathrm{~A})$ to an element $y \in \operatorname{Supp}(f)$. So we must have, the $a_{i}$ being conjugation-invariant:

$$
\left|a_{i}(\gamma)\right|=\left|a_{i}(y)\right|<1 .
$$

However, $a_{i}(\gamma) \in \mathbb{Q}$, and the product formula gives $\left|a_{i}(\gamma)\right|=1$ if $a_{i}(\gamma) \neq 0$. Thus $a_{i}(\gamma)=0$ for all $i$, which means that $\gamma$ is unipotent.

Assume now that $K_{S}$ satisfies the assumption of Lemma 5: then we get, by Lemma 4:

$$
\text { trace } r_{\text {cusp }}(f)=J_{\mathbf{v}_{0}}(f)=\int_{G(\mathbb{Q}) \backslash G(\mathbb{A})}\left\{\sum_{\gamma \in \mathbf{D}_{0}} f\left(x^{-1} \gamma x\right)\right\} d x
$$

where $\mathfrak{v}_{0}$ is now the set of all unipotent elements of $G(\mathbb{Q})$.

We now use a fundamental result of Arthur about the distribution $J_{\mathrm{o}_{0}}$ associated to $\mathfrak{b}_{0}$, the unipotent orbit. We denote it by $J_{\text {unip }}$. Recall that, for general $f$, the distribution $J_{\text {unip }}^{T}$ depends on a parameter $T$ (only for our particular choice of $f$ is it independent of $T$ ). For a certain choice $T_{0}$ of $T, J_{\text {unip }}^{T}$ has particularly nice properties. We will denote by $J_{\text {unip }}(f)$ the distribution $J_{\text {unip }}^{T_{O}}(f)$ (see $\left.[5, \S 1] ;[4, \S 1-2]\right)$.

Recall that the subgroup $K^{S}$ of $G^{S}$ defined before the statement of Theorem $1 \mathrm{~A}$ is fixed. For simplicity we will assume that $K^{S}$ is maximal compact in $G^{S}$, a product of maximal-compact local subgroups. (It is easy to see that the following argument does not really depend on that assumption).

Let $S^{\prime}=S \cup\left\{p_{0}\right\} \cup\{\infty\}$. Let $\mathscr{H}_{S^{\prime}}$ be the Hecke algebra of functions on $G(\mathbb{A})$ which are finite sums of functions of the form $g_{S^{\prime}} \otimes f^{S^{\prime}}$, where $g_{v} \in C_{c}^{\infty}\left(G\left(\mathbb{Q}_{v}\right)\right)$ for $v \in S^{\prime}$, and $f^{S^{\prime}}$ is as above. We write $G\left(\mathbb{Q}_{S^{\prime}}\right)=\prod_{v \in S^{\prime}} G\left(\mathbb{Q}_{v}\right)$. Thus $\mathscr{H}_{S^{\prime}}$ is the space of functions on $G(\mathbb{A})$ which are unramified outside $S^{\prime}$.

Let $\mathscr{U}_{S^{\prime}}$ be the set of unipotent conjugacy classes of $G_{S^{\prime}}$ : it is finite. If $U$ is a unipotent conjugacy class in $G(\mathbb{Q})$, the product $U_{S^{\prime}}$ of its points with values in the local fields is a unipotent class in $\mathscr{U}_{S^{\prime}}$. Let $\mathscr{U}_{S^{\prime}, \mathbb{Q}}$ be the subset of $\mathscr{U}_{S^{\prime}}$ defined by such classes.

Theorem 2 (Arthur $[5,6]$ ). (1) For each $U \in \mathscr{U}_{S^{\prime}, \mathbb{Q}}$ there is a measure $m_{U}$ on $U$ such that, for any $f \in \mathscr{H}_{\mathrm{s}^{\prime}}$ :

$$
J_{\text {unip }}(f)=\sum_{U \in \mathscr{U}_{S^{\prime}, \mathbb{Q}}} \int_{U} f(x) d m_{U}(x) .
$$

(2) The measure $m_{U}$ is absolutely continuous with respect to the measure on $U$ invariant by $G_{S^{\prime}}$.

(3) For the unit orbit $U=\{1\}$ we have

$$
m_{U}(f)=\operatorname{vol}(G(\mathbb{Q}) \backslash G(\mathbb{A})) f(1) .
$$


This follows from the following results: Fix a minimal parabolic subgroup $P_{0}=M_{0} N_{0}$ of $G$ over $\mathbb{Q}$. For any Levi subgroup $M$ of $G$ containing $M_{0}$, and unipotent conjugacy class $U \subset G\left(\mathbb{Q}_{S^{\prime}}\right)$, Arthur defines in [6, Thm. 5.2] a distribution $J_{M}(U, f)$ on $C_{c}^{\infty}\left(G\left(\mathbb{Q}_{s^{\prime}}\right)\right.$ ). (It is a limit of weighted orbital integrals on semi-simple elements.) It is shown to be absolutely continuous with respect to the invariant measure on the orbit [6, Thm. 5.2]. Moreover, in [5, Cor. 8.3] it is shown that

$$
J_{\text {unip }}(f)=\sum_{M} \sum_{U \in \mathscr{\mathscr { U }}_{S^{\prime}, \mathbb{Q}}} a^{M}(U) J_{M}(U, f) ;
$$

the $a^{M}(U)$ are certain constants, $M$ ranges over Levi subgroups as before. Finally, the constant for $U=\{1\}$ is computed in [5, Cor. 8.5].

We now apply Theorem 2 to $J_{\text {unip }}(f)$, with $f$ as before. Recall that, at the places of $S$, we have $f_{S}=\operatorname{vol}\left(K_{S}\right)^{-1} h_{S}, h_{S}$ the characteristic function of $K_{S}$. We have:

$$
J_{\text {unip }}(f)=\operatorname{vol}(G(\mathbb{Q}) \backslash G(\mathbb{A})) f(1)+\sum_{U \neq 1} \int_{U} f(x) d m_{U}(x) .
$$

Writing $v$ for $\operatorname{vol}(G(\mathbb{Q}) \backslash G(\mathbb{A}))$, this yields:

$$
\begin{aligned}
& \operatorname{vol}\left(K_{S}\right) J_{\text {unip }}(f)=v \cdot f_{\infty}(1) \cdot v\left(K^{S}\right)^{-1} \\
& \quad+\sum_{U \neq 1} \int_{U_{\infty} \times U_{0} \times U_{S}} f_{\infty}\left(x_{\infty}\right) f_{0}\left(x_{0}\right) \cdot h_{S}\left(x_{S}\right) d m_{U}\left(x_{\infty}, x_{0}, x_{S}\right) . \\
& \quad\left(\text { Recall that } f_{0}(1)=1 .\right)
\end{aligned}
$$

Since $U$ is the product of local orbits associated to a rational orbit, we have $U_{p} \neq\{1\}$ for $p \in S$.

Lemma 6. Let $U$ be a unipotent orbit $\neq\{1\}$ in the reductive p-adic group $G$. Then, if $g_{K}$ is the characteristic function of the compact-open subgroup $K$ :

$$
\lim _{K \rightarrow 1} \int_{U} g_{K}(x) d x=0
$$

where $d x$ is the invariant measure on $U$.

This is obvious by Lebesgue's dominated convergence theorem: the integral is absolutely convergent, by a well-known result of Rao and Deligne. It is majorized, say, by the integral of $g_{K_{0}}$ for $K_{0}$ fixed, and $g_{K}(x) \rightarrow 0$ pointwise when $K \rightarrow 1$.

But now, using absolute continuity of the measure $m_{U}$ with respect to the invariant measure on $U$, Lemma 6 and Fubini's Theorem imply that each of the terms associated to $U \neq 1$ in the expression $(* *)$ tends to 0 when $K_{S} \rightarrow 1$. So we have proved:

\section{Lemma 7.}

$$
\lim _{K_{S} \rightarrow 1} \operatorname{vol}\left(K_{S}\right) J_{\text {unip }}(f)=\operatorname{vol}(G(\mathbb{Q}) \backslash G(\mathbb{A})) \cdot f_{\infty}(1) \cdot v\left(K^{S}\right)^{-1}
$$


We know (cf. after the proof of Lemma 5) that $J_{\text {unip }}(f)=\operatorname{trace} r_{\text {cusp }}(f)$. We now analyze this trace using the speciàl properties of $f$. At the places $p \neq p_{0}, f_{p}$ is just the projector associated to $K_{p}$. Thus, writing as before $\mathscr{L}$ for $L_{\text {cusp }}^{2}(G(\mathbb{Q}) \backslash G(\mathbb{A}))$ :

$$
\operatorname{trace} r_{\text {cusp }}(f)=\operatorname{trace}\left(f_{\infty} \otimes f_{0} \mid \mathscr{L}^{K_{S} K^{S, p_{0}}}\right)
$$

where $\mathscr{L}^{K}$ denotes the vectors of $\mathscr{L}$ fixed by a group $K$. (Note that $\mathscr{L}^{K_{s} K^{S, p_{0}}}$ is a representation of $G_{\infty} \times G_{p_{0}}$ ).

We now use the properties of $f_{\infty}$ and $f_{0}$. By Lemma 1 , trace $\pi\left(f_{\infty}\right) \neq 0$ only for a finite number $\pi_{1, \infty}=\delta, \pi_{2, \infty}, \ldots, \pi_{n, \infty}$ of representations of $G_{\infty}$. Moreover, since $\pi_{0}$ is a supercuspidal representation of $G_{p_{0}}$, we have trace $\sigma\left(f_{0}\right)=0$ for any irreducible representation $\sigma \neq \pi_{0}$ of $G_{p_{0}}$; and by our choice of $f_{0}$, trace $\pi_{0}\left(f_{0}\right)=\frac{1}{d\left(\pi_{0}\right)}$. This allows us to rewrite:

$$
\text { trace } r_{\text {cusp }}(f)=\frac{1}{d\left(\pi_{0}\right)} \sum_{i=1}^{n} \operatorname{trace} \pi_{i, \infty}\left(\mathrm{f}_{\infty}\right) \mathrm{m}\left(\pi_{i, \infty} \otimes \pi_{0}, \mathscr{L}^{K_{S} K^{S, p_{0}}}\right)
$$

where $m(\pi, \mathscr{V})$ is the multiplicity of $\pi$ in a representation $\mathscr{V}$.

We will now use the following lemma, which will be proved later:

Lemma 8. Assume $\pi_{i, \infty} \neq \delta$ (so $i \neq 1$ ). Then

$$
\lim _{K_{S} \rightarrow 1} v\left(K_{S}\right) m\left(\pi_{i, \infty} \otimes \pi_{0}, \mathscr{L}^{K_{S} K^{S, p_{0}}}\right)=0
$$

Combining now Lemmas 7 and 8 , the identity $J_{\text {unip }}(f)=\operatorname{trace} r_{\text {cusp }}(f)$ and the expression for trace $r_{\text {cusp }}(f)$, we get, using that trace $\delta\left(f_{\infty}\right)=1$ :

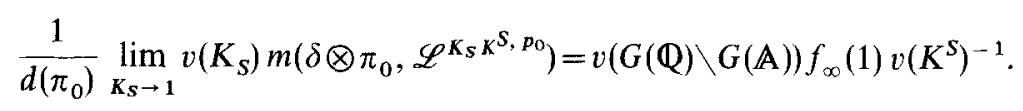

We still need a last lemma:

Lemma 9. $f_{\infty}(1)=d(\delta)$, the formal degree of $\delta$.

Proof. This is well-known and follows immediately from the Plancherel formula (see Lemma 12, where the $p$-adic case is detailed).

We now notice that the multiplicity of $\delta$ in the space $\mathscr{L}^{K_{0} K_{S} K^{S}, p_{0}}$ is at least $\operatorname{dim}\left(\pi_{0}^{K_{0}}\right)$ multiplied by $m\left(\delta \otimes \pi_{0}, \mathscr{L}^{K_{s} K^{S, p_{0}}}\right)$. Thus:

$$
\begin{aligned}
\liminf _{K_{S} \rightarrow 1} v\left(K_{S}\right) m\left(\delta, \mathscr{L}^{K_{0} K_{S} K^{S, p_{0}}}\right) \\
\quad \geqq \operatorname{dim}\left(\pi_{0}^{K_{0}}\right) \lim _{K_{S \rightarrow 1}} v\left(K_{S}\right) m\left(\delta \otimes \pi_{0}, \mathscr{L}^{K_{S} \boldsymbol{K}^{S, p_{0}}}\right) \\
\quad \geqq d\left(\pi_{0}\right) \operatorname{dim}\left(\pi_{0}^{K_{0}}\right) v(G(\mathbb{Q}) \backslash G(\mathbb{A})) d(\delta) v\left(K^{S}\right)^{-\mathbf{1}} .
\end{aligned}
$$

This is the statement of Theorem $1 \mathrm{~A}$. 
3.3. We still have to prove Lemma 8 ; the proof relies on the method of DeGeorge-Wallach (see also [31]). For simplicity we just write $\pi$ for $\pi_{i, \infty}$; thus $\pi$ does not belong to the discrete series. Remark that by the usual properties of $\pi_{0}$ and $f_{0}$, the multiplicity of $\pi_{0}$ in a $G_{p_{0}}$-space $\mathscr{V}$ is equal to $d\left(\pi_{0}\right) \operatorname{trace}\left(f_{0} \mid \mathscr{V}\right)$. We will use that without comment.

We will now apply the trace formula to a function $f=f_{\infty} \otimes \underset{p \neq \infty}{\otimes} f_{p}$; the $f_{p}$ associated to the finite primes are as above, but the function $f_{\infty}=u$ will be a new function, to be defined later.

In $G_{\alpha}$, let us denote by $B_{r}$ the ball of radius $r>0$ : writing a Cartan decomposition $G_{\infty}=K_{\infty} A^{+} K_{\infty}, B_{r}$ is the set of all $g=k_{1} a k_{2}$ such that $a=\exp (H)$ where $H \in \mathfrak{a}=\operatorname{Lie} A$ has length $\|H\|<r$ for the Killing metric (cf. $[20, \S 2])$.

Lemma 10. (i) For each compact-open $K_{S} \subset G_{S}$, there is $r>0$ with the following property. Let $f=u \otimes \underset{p \neq \infty}{\bigotimes} f_{p}$, with $f_{p}$ as before and $u \in C_{c}^{\infty}\left(G_{\infty}\right)$. Then, if $\operatorname{Supp}(u)$ $\subset B_{r}, f\left(x^{-1} \gamma x\right)=0$ for any $x \in G(\mathbb{A})$ and $\gamma \in G(\mathbb{Q})$ unless $\gamma$ is unipotent.

(ii) Let $r\left(K_{S}\right)$ be the maximum such $r$. Then, as $K_{S} \rightarrow 1, r\left(K_{S}\right) \rightarrow \infty$.

Proof. We use the notations of Lemma 5 and its proof. The proof shows that we will have $f\left(x^{-1} \gamma x\right)=0$ unless $\gamma$ is unipotent, provided

Writing

$$
\left|a_{i}(x)\right|<1 \quad \text { for all } x \in \operatorname{Supp}(f) .
$$

$$
\left|a_{i}(x)\right|=\left|a_{i}\left(x_{\infty}\right)\right|_{\infty} \prod_{p \in S}\left|a_{i}\left(x_{p}\right)\right|_{p} \prod_{p \notin S}\left|a_{i}\left(x_{p}\right)\right|_{p},
$$

we see that this can be ensured by bounding the support of $u$, proving (i). As $K_{S} \rightarrow 1$, the maximum value of the middle term on $K_{S}$ tends to 0 ; thus $\left|a_{i}\left(x_{\infty}\right)\right|_{\infty}$ can be made as large as we want, proving (ii).

We now fix $K_{S}$. If $\operatorname{Supp}(u) \subset B_{r}$, where $r=r\left(K_{S}\right)$, the conclusion of Lemma 5 is still valid; using, as before, Theorem 2, we obtain the expression (cf. formula (**) after Lemma 7)

$$
\begin{aligned}
& v\left(K_{S}\right) \operatorname{trace}\left(u \otimes f_{0} \mid \mathscr{L}^{K_{S} K^{S, p_{0}}}\right)=v \cdot u(1) v\left(K^{S}\right)^{-1} \\
& \quad+\sum_{U \neq 1} \int_{U_{\infty} \times U_{0} \times U_{S}} u\left(x_{\infty}\right) f_{0}\left(x_{0}\right) h_{S}\left(x_{S}\right) d m_{U}(x) .
\end{aligned}
$$

We now apply this formula to a function $u=w * \tilde{w}$ (convolution product) where $w \in C_{c}^{\infty}\left(G_{\infty}\right)$ and $\tilde{w}(g)=\bar{w}\left(g^{-1}\right)$. The representation of $G_{\infty}$ which appears in the left-hand side of our formula is trace-class; in any trace-class representation $\rho$ we have:

$$
\operatorname{trace} \rho(u)=\|\rho(w)\|_{2}^{2}
$$

where \|\|$_{2}$ denotes the Hilbert-Schmidt norm of operators. Moreover, $u(1)$ $=\|w\|_{2}^{2}$, the $L^{2}$-norm on $G_{\infty}$.

Recall that $f_{0}(g)=\langle g w, w\rangle$ where $w$ is in the space of $\pi_{0}$. In any irreducible representation $\sigma_{0}$ of $G_{p_{0}}$, of type $\pi_{0}, \sigma_{0}\left(f_{0}\right)$ projects on a well-defined one- 
dimensional subspace, the image of $\mathbb{C} w$ by the isomorphism $\pi_{0} \cong \sigma_{0}$. Let us denote by $\rho$ the representation of $G_{\infty}$ on the subspace of $\mathscr{L}^{K_{S}, K^{S, p_{0}}}$ thus defined. We have

$$
\begin{aligned}
v\left(K_{S}\right)\|\rho(w)\|_{2}^{2}= & v \cdot\|w\|_{2}^{2} \cdot v\left(K^{S}\right)^{-1} \\
& +\sum_{U} \int_{U_{\infty} \times U_{0} \times U_{S}}(w * \tilde{w})\left(x_{\infty}\right) f_{0}\left(x_{0}\right) h_{S}\left(x_{S}\right) d m_{U}(x) .
\end{aligned}
$$

We apply this to the following function $w$ : let $e$ be a unit vector in the space of $\pi$ and $f(g)=\langle g e, e\rangle$ the associated coefficient. Set $w=h f$, where $h$ is a smooth approximation to the characteristic function of $B_{r / 2}$. (Note that the convolution of $B_{r / 2}$ by itself is contained in $B_{r}-$ cf. [33, p. 320]). We then get:

$$
\begin{aligned}
v\left(K_{S}\right) m(\pi, \rho)\|\pi(w)\|_{2}^{2} & \leqq v\left(K_{S}\right)\|\rho(w)\|_{2}^{2} \\
& \leqq v \cdot\|w\|_{2}^{2} v\left(K^{S}\right)^{-1}+\sum_{U} \int(w * \tilde{w})\left(x_{\infty}\right) f_{0}\left(x_{0}\right) h_{S}\left(x_{S}\right) d m_{U}(x) .
\end{aligned}
$$

If now $h$ tends to the characteristic function $h_{r / 2}$ of $B_{r / 2}, w$ tends to a compactly supported function; $\pi(w)$ is still a Hilbert-Schmidt operator; it is easy to see that all the terms in the inequality tend continuously to their values for $w=h_{r / 2} f$, and thus the inequality is preserved for $w$.

Lemma 11. For $w=h_{r / 2} f,\|\pi(w)\|_{2}^{2} \geqq\|w\|_{2}^{4}$.

This is proved in [20]: since $\bar{f}$ is a matrix coefficient,

$$
\|\pi(w)\|_{2}^{2} \geqq\left|\int \bar{f}(g) w(g) d g\right|^{2}=\left.\left.\left|\int_{B_{r / 2}}\right| w(g)\right|^{2} d g\right|^{2}=\|w\|_{2}^{4} .
$$

Using Lemma 11 and the previous inequality for $w$, we get:

$$
v\left(K_{S}\right) m(\pi, \rho) \leqq c\|w\|_{2}^{-2}+\|w\|_{2}^{-4} \sum_{U} \int(w * \tilde{w})\left(x_{\infty}\right) f_{0}\left(x_{0}\right) h_{S}\left(x_{S}\right) \cdot d m_{U}(x),
$$

where $c$ is a constant independent of $K_{S}$. Note that the inequality holds, in fact, for any $w=h_{r} f, f$ being our coefficient and $h_{r}$ the characteristic function of $B_{r}$ with $r<\frac{1}{2} r(K)$.

Let us adopt this choice of $w$. Since $w * \tilde{w}$ is a function of positive type, we have

$$
|(w * \tilde{w})(x)| \leqq(w * \tilde{w})(1)=\|w\|_{2}^{2}
$$

for any $x \in G_{\infty}$. Moreover, $w * \tilde{w}$ is supported in $B_{2 r}$, so

$$
|w * \tilde{w}(x)| \leqq\|w\|_{2}^{2} h_{2 r}(x) \quad \text { for all } x \in G_{\infty} .
$$

We obtain the inequality:

$$
v\left(K_{S}\right) m(\pi, \rho) \leqq c\|w\|_{2}^{-2}+\|w\|_{2}^{-2} \sum_{U} \int h_{2 r}\left(x_{\infty}\right)\left|f_{0}\left(x_{0}\right)\right| h_{S}\left(x_{S}\right) d v_{U}(x)
$$


where $d v_{U}$ is the absolute value of the measure $d m_{U}$. Now, since $f$ is a coefficient of $\pi$ and $\pi$ is not square-integrable, we have

$$
\int_{G_{\infty}}|f(g)|^{2} d g=\infty
$$

Given $\varepsilon>0$, we may then choose $r$ large enough that, for $w=h_{r} f$, $\|w\|_{2}^{2}>\varepsilon^{-1}$. We then have, if $r\left(K_{S}\right)>2 r$ :

$$
v\left(K_{S}\right) m(\pi, \rho) \leqq \varepsilon\left\{c+\sum_{U} \int h_{2 r}\left(x_{\infty}\right)\left|f_{0}\left(x_{0}\right)\right| h_{S}\left(x_{S}\right) d v_{U}(x)\right\} .
$$

Consider now a sequence of $K_{S} \rightarrow 1$. For $K_{S}$ small enough, $r\left(K_{S}\right)>2 r$; so this inequality is satisfied. Moreover, the functions $h_{S}$ decrease to 0 . An application of Lebesgue's theorem, using the absolute continuity of $d v_{U}$ with respect to the invariant measure on $U$ (cf. after Lemma 6) then shows that each of the terms in the sum indexed by $U$ tends to 0 . So we have:

$$
\lim _{K_{S} \rightarrow 1}\left(v\left(K_{S}\right) m\left(\pi, \rho_{K_{S}}\right) \leqq \varepsilon c .\right.
$$

(We have written $\rho_{K_{s}}$ for the representation previously denoted by $\rho$ to make clear now its dependence on $K_{S}$ ). Since $\varepsilon$ was arbitrary, we have at last:

$$
\lim _{K_{S} \rightarrow 1} v\left(K_{S}\right) m\left(\pi, \rho_{K_{S}}\right)=0
$$

which means, by the definition of $\rho_{K_{S}}$ :

$$
\lim _{K_{S} \rightarrow 1} v\left(K_{S}\right) m\left(\pi \otimes \pi_{0}, \mathscr{L}^{K_{S} K^{S, p_{0}}}\right)=0 .
$$

This is Lemma 8.

3.4. So far we have assumed that $K^{S, p_{0}}$ was a product of local groups, but this condition was irrelevant: remark that we could arrange this by enlarging $S$. If we do this, however, $K_{S}$ will tend to 1 only at some places of $S$. But it is clear that in the arguments after Theorem 2 , we only needed that $K_{S} \rightarrow 1$ at some (non-empty) set of places of $S$. This terminates the proof of Theorem $1 \mathrm{~A}$.

\section{Extension to the finite primes}

4.1. In this paragraph, we state an extension of the adelic theorem to discrete series representations of $G\left(\mathbb{A}_{S}\right)=\prod_{v \in S} G\left(\mathbb{Q}_{v}\right)$, where $S$ is a finite set of places of $\mathbb{Q}$ containing the infinite prime. The proof given in $\S 3$ applies without any transformation; however, we will need the existence of pseudo-coefficients for discrete series representations of $p$-adic groups. Fortunately, this is an easy consequence of the invariant Paley-Wiener theorem for $p$-adic groups recently proved by Bernstein, Deligne and Kazhdan. 
4.2. We will first state the Paley-Wiener theorem of [9]. For this, let us denote by $G$ a reductive $p$-adic group. Let $R(G)$ be the Grothendieck group of admissible $G$-modules of finite length, $\operatorname{Irr}(G)$ the set of irreducible representations of $G$. The set $\operatorname{Irr}(G)$ has a natural decomposition into connected components; each component is associated to a cuspidal datum $\Omega=(M, \rho)$ where $M$ is a Levi subgroup of $G$ and $\rho$ a supercuspidal, irreducible $M$ module.

If $M$ is a Levi subgroup of $G$, the unramified, one-dimensional characters of $M$ form a complex torus, denoted by $\Psi(M)$. If $\omega$ is a representation of $M$ and $\chi \in \Psi(M)$ we may form the representation $\omega \otimes \chi$.

Let $\lambda$ be an additive functional: $R(G) \rightarrow \mathbb{C} ; \lambda$ is called good if

(i) The function $\lambda: \operatorname{Irr}(G) \rightarrow \mathbb{C}$ is supported on a finite number of components.

(ii) For any proper Levi subgroup $M$, and $\sigma \in R(M)$, the function $\chi \mapsto \lambda\left(i_{G M}(\sigma \otimes \chi)\right)$ is a regular function on the variety $\Psi(M)$.

(Here we denote by $i_{G M}(\sigma \otimes \chi)$ the representation induced from $P=M N$ to $G$ of $\sigma \otimes \chi$, where $P=M N$ is any parabolic subgroup having $M$ as a Levi component).

Theorem 3 (Bernstein-Deligne-Kazhdan). Let $\lambda: R(G) \rightarrow \mathbb{C}$ be an additive functional. Then $\lambda$ is good if and only if there is a function $f \in C_{c}^{\infty}(G)$ such that

for any $\pi \in R(G)$.

$$
\lambda(\pi)=\text { trace } \pi(f)
$$

We will deduce from this (we assume, for simplicity, that $G$ is now semisimple):

Proposition 1. Let $\delta$ be a discrete series representation of $G$. Then there is a function $f \in C_{c}^{\infty}(G)$ such that

(1) trace $\delta(f)=1$,

(2) trace $\pi(f)=0$ for any tempered irreducible representation $\pi \neq \delta$ of $G$.

Before proving Proposition 1, we need a few preliminaries about the Langlands classification for $p$-adic groups (Silberger [32], Borel-Wallach [14]). We refer to [14] for details. Choose a minimal parabolic subgroup $P_{0}=M_{0} N_{0}$ of $G$. If $P=M N$ is standard, let $A=A_{M}$ be the split component of $M$, a its "real Lie algebra". Let $a \mapsto H(a)$ be the Harish-Chandra map: $A \rightarrow \mathrm{a}$.

We say that a representation $\sigma$ of $M$ is essentially tempered if there is a character $\chi$ of $M$ such that $\sigma \otimes \chi$ is tempered. If $\sigma$ is an irreducible representation of $M$, and $\omega_{\sigma}$ its central character, we define $\lambda_{\sigma} \in \mathfrak{a}^{*}$ by $\log \left|\omega_{\sigma}(a)\right|$ $=\left\langle\lambda_{\sigma}, H(a)\right\rangle$. We say that $\sigma$ is positive if $\left.\left\langle\lambda_{\sigma}, \alpha\right\rangle\right\rangle 0$ for any root $\alpha$ of $(P, A)$. Langlands' classification theorem then asserts that, if $\sigma$ is essentially tempered and positive, the induced representation $I_{P}(\sigma)$ has a unique irreducible submodule $J_{P}(\sigma)$. Moreover, any irreducible representation of $G$ is so obtained, and $J_{P}(\sigma)$ is equivalent to $J_{p^{\prime}}\left(\sigma^{\prime}\right)$ if and only if $p=p^{\prime}, \sigma=\sigma^{\prime}$.

The following fact is well known to experts. We call a character $I_{p}(\sigma)$, with $\sigma$ positive for $P$, a standard character. 
Proposition 2. The standard characters form a basis over $\mathbb{Z}$ of the Grothendieck group $R(G)$.

Proof. We first show (cf. [31]) that an irreducible representation $\pi$ can be written as a combination of standard characters. Such a representation can be realized as a Langlands submodule $J_{P}(\sigma)$. Moreover, if $\pi^{\prime}$ is another irreducible submodule of $I_{p}(\sigma)$, and $\left(P^{\prime}, \sigma^{\prime}\right)$ are its Langlands data, $\lambda_{\sigma},<\lambda_{\sigma}$ for the natural ordering on $\mathfrak{a}^{*}([14$, Lemma 2.13 , Ch. XI $])$. Moreover, by a theorem of Bernstein-Zelevinski and Casselman $[10,15] \pi^{\prime}$ must come from the same cuspidal datum as $\pi$. This leaves a finite number of possibilities for $\pi^{\prime}$. By induction on $\lambda_{\pi}$, we may assume that any $\pi^{\prime}$ is a combination of standard characters; the same is true for $\pi=J_{p}(\sigma)=I_{p}(\sigma)-\sum \pi^{\prime}$.

We still have to show that the standard characters are independent. Let us fix a cuspidal datum $\Omega=(M, \rho)$. The previous argument shows that all irreducible characters attached to $\Omega$ are linear combinations of standard characters attached to $\Omega$; and the (finite) matrix so obtained is a square, unipotent matrix. Since irreducible characters are independent, this shows that standard characters are in fact independent over $\mathbb{C}$.

We can now prove Proposition 1. Using Proposition 2, we may define a linear functional $\lambda$ on $R(G)$ by setting $\lambda(\delta)=1$ (note that $\delta$ is a standard character!) and $\lambda(\pi)=0$ for any standard character $\pi \neq \delta$. Since a tempered, irreducible representation of $G$ is standard by definition, Proposition 1 will be proved if we can show that $\lambda$ is represented by a function $f$. We must check that $\lambda$ is a good functional. Condition (i) is satisfied; we will check that $\lambda$ vanishes on any induced representation $\pi=I_{M N}^{G}(\tau)$ with $M \neq G$. Using again Proposition 2, we may assume that $\tau$ is a standard representation of $M: \tau$ $=I_{M^{\prime}\left(N^{\prime} \cap M\right)}^{M} \sigma, \sigma$ an essentially tempered representation of $M^{\prime}$, positive for $\left(N \cap M^{\prime}\right)$. But then $\pi=I_{M^{\prime} N^{\prime}}^{G} \sigma$; if $P_{\sigma}$ is the parabolic subgroup of $G$ containing $M^{\prime}$ such that the roots of $\lambda_{\sigma}$ with respect to $A^{\prime}$ are determined by $\left\langle\lambda_{\sigma}, \alpha\right\rangle<0, \pi$ is then a standard representation associated to $\left(\sigma, P_{\sigma}\right)$. $\left(P_{\sigma}\right.$ may not be standard; however, conjugating it to a standard parabolic subgroup reduces the situation to a standard one). Thus $\lambda(\pi)=0$. This obviously implies condition (ii), showing that $\lambda$ is a good functional. Theorem 3 then implies Proposition 1.

4.3. We now revert to the assumptions and notations at the beginning of $\S 3.1$. Let $S$ be a finite set of places of $\mathbb{Q}$ containing $\infty$. Let $p_{0}$ be a prime such that $G_{p_{0}}$ has supercuspidal representations. We choose $\pi_{0}, K_{0}$ as in 3.1.

Let $S^{\prime}$ be a finite set of (finite) primes disjoint of $S$ and $\left\{p_{0}\right\}$. We define $K_{S^{\prime}}$ as in $\S 3.1$, replacing $S$ by $S^{\prime}$. As in $\$ 3.1$, we also choose global and local measures. We assume chosen a compact-open subgroup $K$ of $\prod_{p \notin S \cup S^{\prime} \cup\left\{p_{0}\right\}} G_{p}$. Again, $\mathscr{L}=L_{\text {cusp }}^{2}(G(\mathbb{Q}) \backslash G(\mathbb{A})) ; \mathscr{L}^{K_{0} \times K_{S^{\prime}} \times \mathbf{K}}$ is now a representation of $G_{S}$.

Let $\delta_{S}$ be a discrete series representation of $G_{S}$ : thus $\delta_{S}=\bigotimes_{v \in S} \delta_{v}$ where, for all $v, \delta_{v}$ is a discrete series representation of the real or $p$-adic group $G_{v}$.

Theorem $1 \mathrm{~B}$.

$$
\liminf _{K_{S^{\prime} \rightarrow 1}}\left[v\left(K_{S^{\prime}}\right) \operatorname{mult}\left(\delta_{S}, \mathscr{L}^{K_{0} \times K_{S^{\prime}} \times K}\right)\right] \geqq c
$$


with

$$
c=v(G(\mathbb{Q}) \backslash G(\mathbb{A})) d\left(\pi_{0}\right) \operatorname{dim}\left(\pi_{0}^{K_{0}}\right) v(K)^{-1} d\left(\delta_{S}\right) .
$$

The proof is the same as for Theorem 1A, using the function $f_{S}=\otimes f_{v}$ where $f_{v}$ is a pseudo-coefficient of $\delta_{v}$ as in Proposition 1 . We need only check for $p$ adic groups the analogues of Lemma 1 and Lemma 9. Lemma 1 is obvious: if $f_{v}$ is a pseudo-coefficient of $\delta_{v}$, and trace $\pi_{v}\left(\mathbf{f}_{v}\right) \neq 0$, then by the arguments around Proposition $1, \pi_{v}$ must be attached to the same cuspidal datum as $f_{v}$; this leaves a finite number of possibilities. By the choice of $f_{v}$, moreover, $\pi_{v}$ cannot belong to the discrete series.

We still need the analogue of Lemma 9. (Note that this would be unnecessary if we just wanted to know that Theorem $1 \mathrm{~B}$ holds for some $c>0$ ).

Lemma 12. Let $\delta$ be a discrete series representation of the p-adic group $G$; assume $f \in C_{c}^{\infty}(G)$ satisfies the conditions of Proposition 1. Then

$$
f(1)=d(\delta)
$$

Proof. We use the Plancherel formula, proved by Harish-Chandra [23]. This expresses $f(1)$ as an integral $([23, \S 13])$ :

$$
f(1)=\sum_{M} c_{M} \int_{\mathscr{E}_{2}(M)} d(\omega) \mu(\omega) \operatorname{trace}\left(\pi_{\omega}(f)\right) d \omega
$$

The sum ranges over conjugacy classes of Levi subgroups; $\mathscr{E}_{2}(M)$ is the variety of unitary square integrable representations of $M ; \pi_{\omega}$ is the associated induced representation, $d(\omega)$ the formal degree of $\omega$ and $\mu(\omega) d \omega$ the Plancherel measure, and $c_{M}$ a certain constant. If $M=G, c_{M}=1$ and $\mu(\omega)=1$. For $f$ as in Proposition 1, all terms associated to $\omega \neq \delta$ vanish, whence

$$
f(1)=d(\delta)
$$

We finish this paragraph with two remarks. First, even when $G(\mathbb{R})$ has no discrete series, the same proof will show that a discrete series representation of $G_{S}$, where $S$ is now a finite set of finite primes, imbeds in a global, cuspidal representation of $G(\mathbb{A})$, say $\pi=\otimes \pi_{v}$; of course, in that case $\pi_{\infty}$ cannot be specified. Second, we have worked over $\mathbb{Q}$ for simplicity of notations; obviously the analogue of Theorem $1 \mathrm{~B}$ and the previous remark hold over any number field.

\section{Proof of Theorem 1 and applications}

5.1. We can now prove Theorem 1. Recall the assumptions: $G$ is a reductive group over $\mathbb{Q}$, connected, simply connected, semi-simple and almost simple. We assume that $G=\underline{G}(\mathbb{R})$ is noncompact; $\Gamma$ is a congruence subgroup of $G$. We assume $p_{0}$ chosen so that $\underline{G}\left(\mathbb{Q}_{p_{0}}\right)$ has a supercuspidal representation. We will give a more precise statement than in the Introduction: 
Theorem 1. There exists a subgroup $\Gamma_{0}$ of finite index of $\Gamma$ with the following property. Let $S$ be a finite set of primes, not containing $p_{0}, \Gamma_{n}$ a sequence of

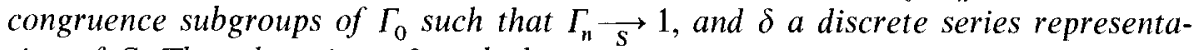
tion of $G$. Then there is $\varepsilon>0$ such that

$$
\liminf _{n \rightarrow \infty} \frac{m\left(\Gamma_{n}, \delta\right)}{v\left(\Gamma_{n} \backslash G\right)}>\varepsilon d(\delta)
$$

with a constant $\varepsilon$ depending only on the limit $K_{p_{0}}$ of the sequence $\left(\Gamma_{n}\right)$ at $p_{0}$.

Proof. Since $\Gamma$ is a congruence subgroup of $G$, we have $\Gamma=G(\mathbb{Q}) \cap K_{\Gamma}, K_{\Gamma}$ a compact-open group in $G\left(\mathbb{A}_{f}\right)$. Let $K^{0}$ be a group admitting a decomposition $K^{0}=K_{p_{0}}^{0} \times K^{0, p_{0}}$ with $K^{0, p_{0}}$ compact-open in $\prod_{p \neq p_{0}} G\left(\mathbb{Q}_{p}\right)$, and contained in $K_{\Gamma}$. We may further assume that $G\left(\mathbb{Q}_{p_{0}}\right)$ has supercuspidal representations with vectors fixed by $K_{p_{0}}^{0}$. We set $\Gamma_{0}=\underline{G}(\mathbb{Q}) \cap K^{0}$.

If now $S$ is a finite set of primes disjoint of $\left\{p_{0}\right\}$ and $\Gamma_{n}$ is as in Theorem 1, let $K^{n}$ be the closure of $\Gamma_{n}$ in $\underline{G}\left(\mathbb{A}_{f}\right)$. Then $K^{n}=K_{p_{0}} \times K_{S}^{n} \times K^{S, p_{0}}$ with $K_{p_{0}}$ fixed and contained in $K_{p_{0}}^{0}$ and $K^{S, p_{0}}$ fixed (at least for large enough $n$ ). We apply Theorem $1 \mathrm{~A}$ to the groups $K^{n}$; we obtain:

$$
\begin{aligned}
& \liminf _{n \rightarrow \infty} v\left(K_{S}^{n}\right) \operatorname{mult}\left(\delta, L_{\text {cusp }}^{2}(\underline{G}(\mathbb{Q}) \backslash \underline{G}(\mathbb{A}))^{K^{n}}\right) \\
& \quad \geqq v(\underline{G}(\mathbb{Q}) \backslash \underline{G}(\mathbb{A})) d\left(\pi_{0}\right) \operatorname{dim}\left(\pi_{0}^{K_{p_{0}}}\right) d(\delta) v\left(K^{S, p_{0}}\right)^{-1} .
\end{aligned}
$$

Strong approximation (cf. [13, $\S 4.3$ and 4.7]) yields an isomorphism of $G$ spaces:

In particular

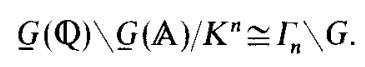

and

$$
L_{\text {cusp }}^{2}(G(\mathbb{Q}) \backslash G(\mathbb{A}))^{K^{n}} \cong L_{\text {cusp }}^{2}\left(\Gamma_{n} \backslash G\right)
$$

$$
\operatorname{vol}\left(K^{n}\right) \operatorname{vol}\left(\Gamma_{n} \backslash G\right)=v(\underline{G}(\mathbb{Q}) \backslash \underline{G}(\mathbb{A})) .
$$

Thus we can rewrite the basic inequality as

$$
\liminf _{n \rightarrow \infty}\left(v\left(\Gamma_{n} \backslash G\right)^{-1} m\left(\delta, \Gamma_{n}\right)\right) \geqq d\left(\pi_{0}\right) \operatorname{dim}\left(\pi_{0}^{\left.K_{p_{0}}\right)} v\left(K_{p_{0}}\right)^{-1} d(\delta) .\right.
$$

This implies Theorem 1.

Remark. Note that we get an apparently stronger result, replacing the multiplicities in the discrete spectrum by the multiplicities in the cuspidal spectrum. By a theorem of Wallach [34], however, they coincide.

5.2. We will now prove a weak result for general arithmetic groups.

Proposition 3. Let $G$ be a connected semi-simple Lie group, $\Gamma$ an arithmetic subgroup of $G$. Then there is a sequence $\Gamma_{n}$ of subgroups of $\Gamma$, of finite index, such 
that, if $\delta$ is a discrete series representation of $G$ :

for a fixed constant $\varepsilon$.

$$
\liminf _{n \rightarrow \infty} \frac{m\left(\delta, \Gamma_{n}\right)}{v\left(\Gamma_{n} \backslash G\right)}>\varepsilon d(\delta)
$$

Proof. We use a series of reductions:

A. Assume that $G=G(\mathbb{R})$ where $G$ is a $\mathbb{Q}$-group satisfying the assumptions of Theorem 1, and $\Gamma$ is strongly arithmetic. Then $\Gamma$ is commensurable with a congruence subgroup $\Gamma_{0}$ as in Theorem 1 . If now $\Gamma_{n} \subset \Gamma_{0}$ is as in Theorem 1, we have $L^{2}\left(\Gamma_{n} \backslash G\right) \subset L^{2}\left(\Gamma_{n} \cap \Gamma \backslash G\right)$; moreover, $\Gamma_{n} \cap \Gamma \backslash \Gamma_{n}$ is a subgroup of $\Gamma_{0} \cap \Gamma \backslash \Gamma_{0}$, so the index $\left|\Gamma_{n} \cap \Gamma \backslash \Gamma_{n}\right|$ is bounded; it is easy to see that the groups $\Gamma_{n} \cap \Gamma$ satisfy the assertions of Proposition 3.

B. Assume that $G=G(\mathbb{R})^{+}$with $\underline{G}$ a group over $\mathbb{Q}$, connected and semisimple, with no $\mathbb{Q}$-factor compact at the infinite place. We consider the simplyconnected covering $\underline{G}^{\text {sc }}$ of $\underline{G}$; we have a surjection $G^{\text {sc }}(\mathbb{R}) \rightarrow G ; G^{\text {sc }}$ is a product of groups as in A. By the same set of arguments as in $A$, we deduce the assertion for $\Gamma$ strongly arithmetic in $G$ from the assertion for $\underline{G}^{\text {sc }}$.

C. More generally assume $\Gamma \subset G$ is strongly arithmetic. Again passing to the simply-connected covering, we may assume that $\underline{G}=\underline{G}_{1} \times \ldots \times G_{r}$ (product of $\mathbb{Q}$-groups); assume, e.g., that $G_{i}=\underline{G}_{i}(\mathbb{R})$ is compact for $i \leqq s$. Then $\Gamma$ is commensurable with $\Gamma^{\prime}=\Gamma \cap\left(G_{s+1} \times \ldots \times G_{r}\right)$. It is easy to deduce from $B$ that the pair $\left(G, \Gamma^{\prime}\right)$ verifies Proposition 3. Thus it also holds for $(G, \Gamma)$.

D. Finally, assume $\Gamma \subset G$ is arithmetic in Margulis' sense: thus there exists $G_{1}$ over $\mathbb{Q}$, and an exact sequence

$$
1 \rightarrow U \rightarrow \underline{G}_{1}(\mathbb{R})^{+} \underset{\pi}{\rightarrow} G \rightarrow 1
$$

with $U$ compact, $\Gamma_{1}$ strongly arithmetic in $\underline{G}_{1}(\mathbb{R})^{+}$such that $\pi\left(\Gamma_{1}\right)$ is commensurable with $\Gamma$. We may replace $\Gamma$ by $\Gamma \cap \pi\left(\Gamma_{1}\right)$. If $\delta$ belongs to the discrete series,

$$
\begin{aligned}
& \operatorname{mult}\left(\delta, L^{2}(\Gamma \backslash G)=\operatorname{mult}\left(\delta \circ \pi, L^{2}\left(\pi^{-1} \Gamma \backslash \underline{G}_{1}(\mathbb{R})^{+}\right)\right)\right. \\
& \quad=\operatorname{mult}\left(\delta \circ \pi, L^{2}\left(\Gamma_{1} U \backslash \underline{G}_{1}(\mathbb{R})^{+}\right)\right)=\operatorname{mult}\left(\delta \circ \pi, L^{2}\left(\Gamma_{1} \backslash \underline{G}_{1}(\mathbb{R})^{+}\right)\right) .
\end{aligned}
$$

We may now take a suitable sequence of subgroups $\Gamma_{n}$ of $\Gamma_{1}$; their image in $G$ will satisfy Proposition 3.

5.3. Cohomology of arithmetic groups. For simplicity we will just assume that $G$ satisfies the assumptions of Theorem 1 . We assume that $G=G(\mathbb{R})$ has a discrete series. Let $K$ be a maximal compact subgroup of $G, q=\frac{1}{2} \operatorname{dim}(G / K)$. Let $(\xi, V)$ be a finite-dimensional representation of $G$.

Proposition 4. Let $\Gamma$ be a congruence subgroup of $G$. Let $\Gamma_{0} \subset \Gamma, \Gamma_{n} \subset \Gamma_{0}$ satisfy the assumptions of Theorem 1.

Then, for $n$ large enough,

$$
\operatorname{dim} H^{q}\left(\Gamma_{n}, V\right)>\varepsilon v\left(\Gamma_{n} \backslash G\right)
$$

with a constant $\varepsilon>0$ depending only on $V$ and the limit of the sequence $\left(\Gamma_{n}\right)$ at $p_{0}$. 
Proof. This is now obvious using the results of Borel-Wallach [14]. We use the notations of $[14, \mathrm{II}, \S 5]$. Let $\mu$ be the highest weight of $V^{*}$. Then $\Lambda=\mu+\rho$ is regular and determines a discrete series representation $\delta=\omega_{A}$. By Theorem 5.3 of $[14, \mathrm{II}]$ we have $H^{q}(\mathfrak{g}, k ; \mathscr{H} \otimes V) \cong \mathbb{C}$ where $\mathscr{H}$ is the space of $K$-finite vectors of $\delta$. On the other hand [14, VII, Cor. 2.7] for $\Gamma$ discrete in $G$

$$
H^{q}(\Gamma, V)=H^{q}\left(\mathfrak{g}, k ; C^{\infty}(\Gamma \backslash G) \otimes V\right) .
$$

Moreover, let $L_{\text {cusp }}^{2}(\Gamma \backslash G)^{\infty}$ be the space of $C^{\infty}$ cusp forms. Then, if $L_{\text {cusp }}^{2}(\Gamma \backslash G)$ $=\bigoplus_{\pi \in \hat{G}} m(\pi) \pi$, we have $H^{q}\left(\mathfrak{g}, k ; L_{\text {cusp }}^{2}(\Gamma \backslash G)^{\infty} \otimes V\right)=\oplus m(\pi) H^{q}(\mathfrak{g}, k ; \pi \otimes V)$, a finite sum (cf. [14, VII, Theorem 3.2]). Moreover Borel [11] has shown that the cuspidal cohomology - the cohomology of $L_{\text {cusp }}^{2}(\Gamma \backslash G)^{\infty}$ - injects in the cohomology of $C^{\infty}(\Gamma \backslash G)$. Thus

$$
\operatorname{dim} H^{q}(\Gamma, V) \geqq m(\delta) \operatorname{dim} H^{q}(\mathfrak{g}, k, \delta \otimes V)=m(\delta) ;
$$

recalling that the multiplicity of $\delta$ in $L^{2}$ is the same as its multiplicity in $L_{\text {cusp }}^{2}$, and applying this and Theorem 1 to $\Gamma_{n}$, we see:

$$
\operatorname{dim} H^{q}(\Gamma, V) \geqq \varepsilon v\left(\Gamma_{n} \backslash G\right) .
$$

5.4. $L^{2}$-cohomology of arithmetic varieties. We finish with a footnote to the article of Borel-Casselman [12]. Let $G$ be as in Theorem 1, $\Gamma \subset G$ a congruence subgroup, $V$ a finite-dimensional representation of $G, \tilde{V}$ the associated local system on $\Gamma \backslash X=\Gamma \backslash G / K$. (We refer to [12] for undefined notions and notations.)

Theorem 4 (Borel-Casselman). The space of $L^{2}$-cohomology $H_{(2)}^{*}(\Gamma \backslash X ; \tilde{V})$ is finite-dimensional if no proper cuspidal parabolic subgroup of $G$ contains a Cartan subgroup of a maximal compact subgroup of $G$.

Conversely, Theorem 1 implies:

Proposition 5. Assume that $G$ contains a proper cuspidal parabolic subgroup $P$ $=$ MAN containing a Cartan subgroup of a maximal compact subgroup of $G$. Then, for suitable arithmetic subgroups $\Gamma_{n}$ of $\Gamma$, the space $H_{(2)}^{q_{M}}\left(\Gamma_{n} \backslash X ; \tilde{V}\right)$ is infinite-dimensional. (Here $2 q_{M}$ is the dimension of the symmetric space of $M$.)

Indeed, this is proved in $[12, \S 4.6]$ under the assumption that $L^{2}\left(\Gamma_{n} \cap M M\right)$ contains a suitable discrete series representation; by Theorem 1, it will be the case for deep enough $\Gamma_{n}$.

Acknowledgement. Thanks are due to N. Wallach, for pointing out the problem to me; to J.-P. Labesse, for noticing a gap in an earlier proof; and to J. Arthur, for communicating his results to me - in particular Theorem 2 - in manuscript form. I also thank The Institute for Advanced Study, in Princeton, for its hospitality while this work was done.

\section{References}

1. Arthur, J.: A Trace Formula for Reductive groups I. Duke Math. J. 45, 911-954 (1978)

2. Arthur, J.: A Trace Formula for Reductive groups II. Compos. Math. 40, 87-121 (1980) 
3. Arthur, J.: Eisenstein series and the Trace Formula. Proc. Symp. Pure Math. 33, 1, 253-276 (1979)

4. Arthur, J.: The Trace formula in invariant form. Ann. Math. 114, 1-74 (1981)

5. Arthur, J.: A measure on the unipotent variety (Preprint)

6. Arthur, $J$.: The local behavior of weighted orbital integrals (In preparation)

7. Arthur, J.: A theorem on the Schwartz space of a reductive Lie group. Proc. Natl. Acad. Sci. USA 72, 4718-19 (1975)

8. Barbasch, D., Moscovici, H.: $L^{2}$-index and the Selberg Trace Formula. J. Funct. Anal. 53, 151201 (1983)

9. Bernstein, J., Deligne, P., Kazhdan, D.: Trace Paley-Wiener Theorem for Reductive p-adic groups. (Preprint)

10. Bernstein, I.N., Zelevinski, A.V.: Induced representations of reductive $p$-adic groups I. Ann. Sci. ENS 10, 441-472 (1977)

11. Borel, A.: Regularization theorems in Lie algebra cohomology. Applications. Duke Math. J. 50, 605-623 (1983)

12. Borel, A., Casselman, W.: $L^{2}$-cohomology of locally symmetric manifolds of finite volume. Duke Math. J. 50, 625-647 (1983)

13. Borel, A., Jacquet, H.: Automorphic forms and automorphic representations. Proc. Symp. Pure Math. 33, 1, 189-202 (1979)

14. Borel, A., Wallach, N.: Continuous cohomology, discrete subgroups, and representations of reductive groups. Princeton: Princeton University Press 1980

15. Casselman, W.: Introduction to the theory of admissible representations of $p$-adic groups. (Mimeographed notes)

16. Clozel, L., Delorme, P.: Sur le théorème de Paley-Wiener invariant pour les groupes réductifs réels. C.R.A.S. Paris (To appear)

17. Clozel, L., Delorme, P.: Pseudo-coefficients et cohomologie des groupes réductifs reels. C.R.A.S. Paris (To appear)

18. Clozel, L., Labesse, J.-P., Langlands, R.P.: Morning seminar on the Trace Formula (mimeographed notes). I.A.S., Princeton 1983-84

19. DeGeorge, D.: On a Theorem of Osborne and Warner. J. Funct. Anal. 48, 81-94 (1982)

20. DeGeorge, D., Wallach, N.: Limit formulas for multiplicities in $L^{2}(\Gamma \backslash G)$. Ann. Math. 107, 133$150(1978)$

21. Gèrardin, P.: Construction de Séries discrètes p-adiques. Lect. Notes 462 . Berlin Heidelberg New York: Springer 1975

22. Harish-Chandra: Harmonic Analysis in Reductive p-adic groups. Proc. Symp. Pure Math. 26, 167-192 (1974)

23. Harish-Chandra: The Plancherel formula for reductive $p$-adic groups. In: Collected Papers, vol. 4. Berlin-Heidelberg-New York-Tokyo: Springer 1984

24. Harish-Chandra: Harmonic Analysis on Reductive p-adic Groups. Lect. Notes in Mathematics 162. Berlin Heidelberg New York: Springer 1975

25. Henniart, G.: La Conjecture de Langlands locale pour GL(3). Mém. Soc. Math. Fr. (To appear)

26. Kazhdan, D.: Arithmetic varieties and their fields of quasi-definition. Actes Cong. Intern. Math. 2, 321-325 (1970)

27. Kazhdan, D.: On Arithmetic varieties II. Isr. J. Math. 44, 139-159 (1983)

28. Labesse, J.P.: La formule des traces d'Arthur-Selberg. Seminaire Bourbaki, no. 636 (1984-85)

29. Mischenko, P.: Invariant tempered distributions on the reductive group $G L\left(n, F_{p}\right)$. Thesis, Toronto 1982

30. Osborne, M.S., Warner, G.: The theory of Eisenstein systems. New York: Academic Press 1981

31. Rogawski, J.: Representations of $G L(n)$ and division algebras over a $p$-adic field. Duke Math. J. 50, 161, $196(1983)$

32. Silberger, A.J.: The Langlands Quotient Theorem for p-adic Groups. Math. Ann. 236, 95-104 (1978)

33. Varadarajan, V.S.: Harmonic Analysis on Real Reductive Groups. Lecture Notes in Mathematics 576. Berlin Heidelberg New York: Springer 1977

34. Wallach, N.R.: On the Constant term of a square-integrable automorphic form. Proceedings of the Neptun Conference on Operator algebras and Group representations 1980 